Archives

13 | 1994

Enquêtes

\title{
Une campagne d'Italie, ou l'archéologie médiévale en action
}

\section{Françoise Piponnier}

\section{OpenEdition}

Journals

Édition électronique

URL : http://journals.openedition.org/ccrh/2708

DOI : $10.4000 /$ ccrh. 2708

ISSN : $1760-7906$

\section{Éditeur}

Centre de recherches historiques - EHESS

Édition imprimée

Date de publication : 4 octobre 1994

ISSN : 0990-9141

Référence électronique

Françoise Piponnier, « Une campagne d'Italie, ou l'archéologie médiévale en action », Les Cahiers du Centre de Recherches Historiques [En ligne], 13 | 1994, mis en ligne le 27 février 2009, consulté le 19 avril 2019. URL : http://journals.openedition.org/ccrh/2708; DOI : 10.4000/ccrh.2708

Ce document a été généré automatiquement le 19 avril 2019

Article L.111-1 du Code de la propriété intellectuelle. 


\title{
Une campagne d'Italie, ou l'archéologie médiévale en action
}

\author{
Françoise Piponnier
}

\section{NOTE DE L'AUTEUR}

L'équipe 1993 : Françoise Piponnier, Philippe Bon, Caterina Laganara, Thomas Le Goff, Annik Le Pape, Anna-Lucia Lionetti, Sébastien Minchin, Isabelle.

1 Trois villes à portée de regard : Torremaggiore, San Severo, Lucera, non loin de Foggia, à l'extrême nord des Pouilles. Un éperon, vaste comme le paysage environnant portait encore quelques ruines, il y a douze ans, lors de la première prospection. A sa pointe, des murs émergeaient à peine d'une grosse motte de terre isolée par un fossé en arc de cercle. Les blocs effondrés de ce qui avait été la cathédrale se repéraient au premier coup d'oeil sur l'étendue herbeuse où des ondulations discrètes laissaient deviner la présence de bâtiments enfouis de la cité. Au bord d'un second fossé large d'une trentaine de mètres, une tour carrée dressait encore un étage de maçonnerie en brique et pierre au milieu des vestiges d'un socle beaucoup plus vaste. Au-delà de ce second fossé, un autre espace, plus petit, était parsemé de traces de constructions. Plan parfait de ville médiévale, hiérarchisé, de la zone seigneuriale, à la ville et au faubourg artisanal. Aucune tentative de remise en culture n'était venue perturber le terrain depuis sa désertion.

2 Ces arguments, assez forts pour déterminer des archéologues à intervenir, n'étaient pas les plus décisifs pour les autorités locales. Ici, en effet, plane le souvenir de celui qui fut le dernier grand souverain des Pouilles, l'empereur Frédéric II Hohenstaufen. C'est dans les murs de la forteresse de Fiorentino qu'un chroniqueur place son trépas, un jour de décembre 1250. Italiens et allemands surtout, des visiteurs viennent parfois rompre la solitude des lieux, parcourus seulement par des troupeaux de moutons. Rêvant sans doute aux succès remportés par d'autres grands sites archéologiques et à leurs retombées économiques et culturelles, sensibilisés par l'existence de sociétés historiques et 
archéologiques locales, les édiles de Torremaggiore ont répondu avec enthousiasme, dans un premier temps, au projet lancé par Maria-Stella Calo Mariani professeur à l'Université de Bari. Dans le cadre d'une convention avec l'École Française de Rome, une équipe de l'EHESS, formée au départ de Françoise Piponnier et Patrice Beck et, ultérieurement, Philippe Bon, a été invitée à former, avec M. S. Calo Mariani et Caterina Laganara de l'Université de Bari, l'encadrement d'un chantier, généreusement doté par la commune de Torremaggiore, qui a fonctionné comme école de fouille jusqu'en 1991. Des difficultés politico-économiques, au plan local, ayant tari la principale source de financement, les recherches ont été poursuivies depuis avec les seuls moyens procurés par l'École Française de Rome. Le but, limité, de cette prolongation étant de terminer les sondages les plus riches d'information avant de passer à la publication.

La campagne 1993 devant être consacrée à la zone urbaine, huit archéologues italiens et français se retrouvaient par un beau jour de septembre, avec trois semaines devant eux pour répondre au plus grand nombre possible d'énigmes irrésolues. Que faire?

Sans ouvriers, le dégagement des murs affleurants entrepris pour reconnaitre le plan des bâtiments, le réseau des rues, identifier le(s) mur(s) d'enceinte n'était plus envisageable.

$5 \quad$ Allait-on laisser inachevée la fouille de la petite église? Malgré les regrets de ceux qui pendant trois campagnes ont démêlé l'écheveau complexe de ses réfections et réaménagements, fouillé et dessiné les sépultures qu'elle contenait, le four et le foyer parfaitement conservés destinés à la fonte de cloches, exploré les fosses et trous de poteaux antérieurs à sa construction, il faut s'y résigner.

6 Dans la rue longeant l'église, des sépultures construites et un ossuaire adossés à cet édifice avaient été découverts, mais aussi douze sols successifs de cailloutis damé. Leur absence, près de la maison située en face, laissait prévoir une vaste dépression, en relation sans doute avec l'escalier mis au jour, en profondeur, sous ce bâtiment I.

7 L'importance des trouvailles faites dans l'espace occupé par cette vaste maison inclinait à concentrer tout l'effort sur ce secteur, incomplètement fouillé. Dans les murs correspondant à une dernière phase d'occupation du site, trois sols successifs avec leurs foyers avaient été repérés. Deux sols de terre battue antérieurs à l'édification du bâtiment I étaient ensuite dégagés, associés à des murs dont l'organisation d'ensemble restait indéchiffrable, à des foyers et à des silos. Un remblai de terre argileuse plus important noyait les structures fort bien conservées d'un pressoir revêtu de mortier fin. Enfin, des creusements du roc, des trous de poteaux, l'escalier qui descendait en profondeur se perdre sous le mur nord du bâtiment attestaient une ou plusieurs occupations antérieures.

8 L'un des silos avaient été fouillé dans le bâtiment I et un autre dans la rue. Un autre type de structure excavée, vraisemblablement une citerne, paraissait devoir retenir aussi l'attention. D'autres fosses à ouverture circulaire de faible diamètre étaient apparues, l'une dans le bâtiment II voisin, l'autre appartenait à une occupation antérieure à l'édification du bâtiment I, sous sa pièce sud. Toutefois, nous avons choisi d'explorer celle découverte dans la pièce nord, car c'était là que les superstructures caractéristiques d'une citerne étaient le mieux conservées. Noyées dans un bloc de mortier très dur inclus dans le mur surplombant l'ouverture de la fosse, deux tuiles formaient un conduit fortement incliné de l'extérieur vers l'intérieur, traversant le mur de part en part, et débouchant juste au-dessus et au centre de la fosse. Une partie de la coupole maçonnée qui devait, à l'origine, recouvrir largement la fosse était encore en place. 
9 Certaines des fosses-silos déjà fouillées s'étant révélées creusées sur plus de quatre mètres, l'équipement avait été prévu pour faire face à une exploration encore plus profonde : une chèvre métallique démontable chaque soir, avec poulie et cordes, et des cordes d'alpinisme pour assurer le fouille et éventuellement aider sa remontée. Sur plusieurs mètres, le puits était creusé verticalement à travers les couches géologiques déjà connues depuis la fouille des silos: une table calcaire d'une quarantaine de centimètres d'épaisseur, puis une terre argileuse à galets sur plus de trois mètres. Enfin, était atteinte une argile verte extrêmement compacte. Et c'est dans ce matériau, assurément imperméable qu'était creusée la citerne proprement dite, formant un réservoir d'environ trois mètres de diamètre.

Naturellement, puits et citerne avaient été remblayés avec les matériaux les plus divers : terre, éléments de démolition, cendres plus ou moins riches en objets et céramiques, mais aussi des cadavres de petits animaux domestiques dont les squelettes sont apparus en connexion lors de la fouille.

11 Dans le réservoir de la citerne, la reconnaissance a été poursuivie sur plus de 2,5 $\mathrm{m}$ de profondeur, sans atteindre le fond. A ce moment, il a été décidé d'arrêter une exploration dangereuse, qui dépassait déjà huit mètres au-dessous du niveau primitif du sol.

12 C'est une fouille plus classique, à ciel ouvert, qui a été réalisée dans tout le reste de l'espace situé au sud du petit mur délimitant le pressoir à huile et sa fosse de décantation, rendue difficile cependant par la profondeur des sondages. En effet, les niveaux archéologiques atteints sont partout antérieurs à la construction du bâtiment I. D'abord deux sols de terre argileuse auxquels sont associées une des fosses-silos et l'ouverture d'un puits vertical, sans doute appartenant à une citerne.

13 Ensuite, sous le même remblai de terre argileuse compacte qui recouvrait le pressoir, a surgi un ensemble de murs de pierres, fort abîmés, mais orientés selon les mêmes directions que le pressoir et les structures qui lui sont associées. Comme la stratigraphie observée antérieurement permet d'affirmer que l'installation de la rue avec ses sols de cailloutis est postérieure à l'enfouissement du pressoir, l'hypothèse se confirme d'un réaménagement drastique du site, à une époque que l'analyse des mobiliers et en particulier de la céramique permettra de préciser, avec un changement de l'orientation des bâtiments et sans doute le percement d'une rue selon un nouvel axe.

14 La présence des murs du bâtiment I restaurés, n'a pas permis de reconnaître l'extension vers l'est du bâtiment antérieur mis au jour cette année au sud-est du pressoir. Deux sols intérieurs sont apparus dans l'angle que formaient ses murs, formés d'argile mêlée de mortier, l'un d'eux comportait un foyer de petites dimensions, il s'agissait donc probablement d'une habitation.

15 Ce ne sont pourtant pas les premières traces d'occupation humaine sur le site. Si l'un des murs repose directement sur le roc et ne recouvrait donc aucun vestige préexistant, l'exploration sous le sol de cette habitation précoce a mis en évidence un creusement du rocher, remblayé, de dimensions très supérieures aux ouvertures des silos et des citernes installés ultérieurement. De même, le démontage du mur sud a prouvé que celui-ci recouvrait deux pavements successifs et un remplissage de terre considérable installé dans un autre creusement du roc, lui aussi de grandes dimensions.

Comme dans la citerne, il a fallu interrompre les recherches avant d'avoir pu répondre à toutes les questions posées et en particulier avant de réussir à élucider le genre d'occupation du site auquel correspondent ces creusements, ou l'époque à laquelle ils 
remontent. Avec la fin de la campagne est arrivée aussi l'interdiction (amicale certes) de poursuivre la fouille ; la profondeur atteinte dépasse déjà largement les limites autorisées pour travailler sans étaiement. Ordre est donné aussi de combler nos sondages, selon les règles de l'art, en ne laissant émerger que les murs du dernier en date des bâtiments.

Les témoins ne manquent pas des tout premiers temps de l'occupation du site, ici des trous de poteaux ou des saignées aménagées dans le roc pour y disposer des sablières basses (on faisait donc une large utilisation du bois dans la construction?), là de vastes creusements... leur interprétation, leurs éventuelles corrélations restent encore énigmatiques.
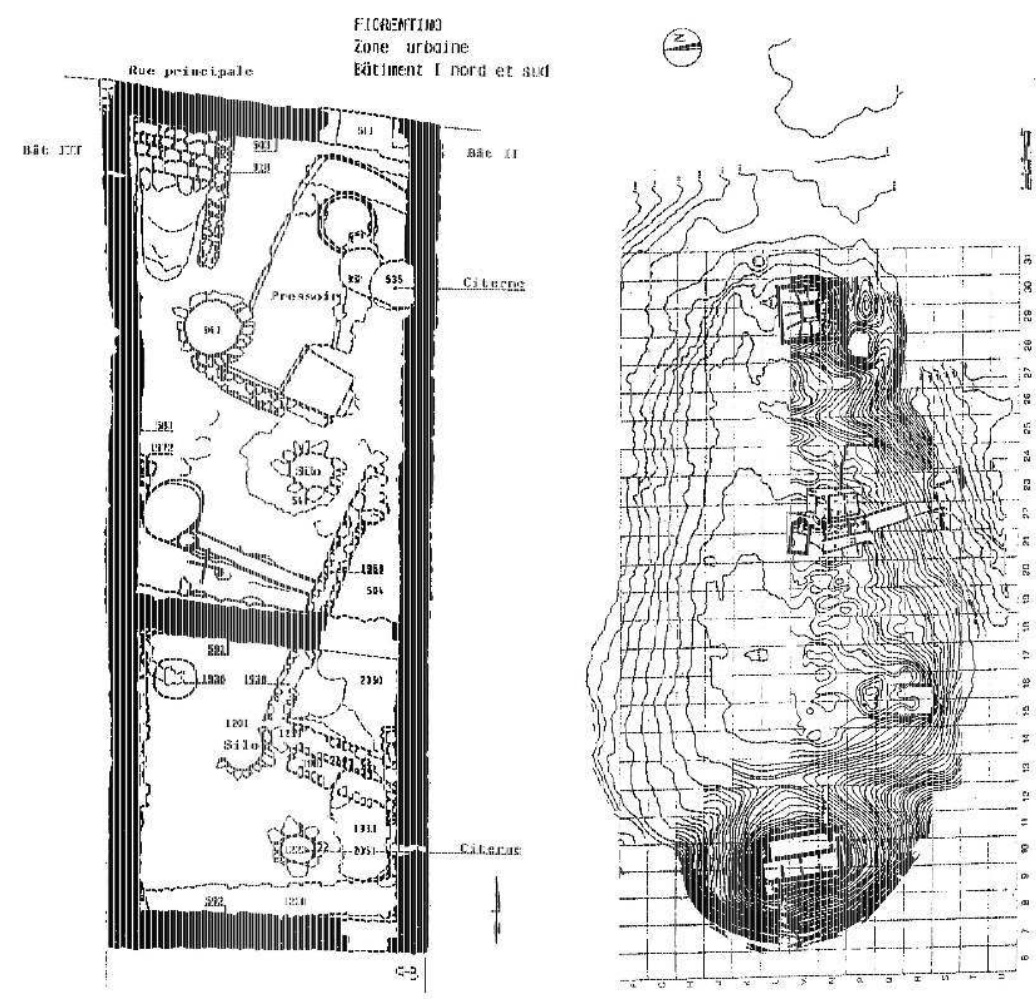